\title{
ORAL MANIFESTATION OF EOSINOPHILIC GRANULOMA: REPORT OF TWO CASES
}

Divya K. S1.

1. Reader, Department Pedodontics, Educare Institute of Dental Sciences, Kottakkal, Kerala.

\section{CORRESPONDING AUTHOR}

Divya K. S.,

Apartment 2A,

Kingswood Apartment,

Bilathikulam, Kozhikode, Pin 673006.

Email-divyaks879@rediffmail.com

\section{HOW TO CITE THIS ARTICLE:}

Divya K. S. "Oral Manifestation of Eosinophilic Granuloma: Report of Two Cases". Journal of Evolution of Medical and Dental Sciences 2013; Vol2, Issue 23, June 10; Page: 4126-4131.

ABSTRACT: Eosinophilic Granuloma (EG) is a rare idiopathic disease characterized by the clonal proliferation of Langerhans cells. EG affects 5 children per million populations. The peak incidence is from one to four years of age. EG involves the head and neck region quite commonly. The first case is of three year old girl who presented to the outpatient department of Pedodontics, with a reddish swelling on the gum in relation to the right upper posterior teeth. She had on examination, soft inflamed gingival enlargement and small ulcers on the mucosa overlying the swelling. Intra oral peri-apical radiograph revealed multiple osteolytic lesions and skull radiograph revealed multiple punched out osteolytic lesions, she further had liver metastasis. The patient was managed using chemotherapy. The second case is of a six year old girl who had a one month history of pain and swelling of the left lower jaw and a day of fever and underwent the removal of badly decayed teeth and was referred in as the swelling persisted even after the procedure. She had no other manifestations of EG and the sole feature was the buccal lesion. The differentials of EG are also discussed. The prognosis of with any other disease depends on early detection. Surgical management alone is used in $50 \%$ of cases, with an additional $23 \%$ of the lesions being treated with both surgery and radiation therapy.

KEYWORDS: Eosinophilic granuloma, Langerhans cell histiocytosis, Histiocytosis X, Gingival lesion.

INTRODUCTION: Eosinophilic Granuloma (EG) or Langerhans cell histiocytosis (LCH) is a rare idiopathic disease characterized by the clonal proliferation of Langerhans cells, which are freely mobile cells that originate from bone marrow myeloid precursors. [1] In 1868, Paul Langerhans discovered epidermal dendritic cells and the conditions which were characterized by these cells were subsequently called different names like Hand-Schuller-Christian disease, Letterer-Siwe disease, and eosinophilic granuloma. Farber (1941) suggested that these three different entities were part of the same disease process with variation in severity, site, and stage. Lichenstein and Jaffe in 1953 included all these entities under the general category of Histiocytosis X. The unification of these three separately described diseases was based on identical histopathologic features of the lesions, which were composed of eosinophils, histiocytes, and lymphocytes. The 
original disease designations, Hand-Schuller-Christian disease, Letterer- Siwe disease, and eosinophilic granuloma, are still used by some practitioners when referring to the clinical features of EG. [2]

EG involves the head and neck region quite commonly, and, in particular, the bones of the skull and jaws. Oral soft tissue lesions are also common and most often found with the gingiva and hard palate. [2] Two cases of EG one involving the deciduous right maxillary first molar in a three year old girl and the other in a six year old girl involving the left lower mandible involving the first and second permanent molars are reported.

CASE REPORT: Case 1: A three year old female presented to the outpatient department of Pedodontics, with a reddish swelling on the gum in relation to the right upper posterior teeth. Her father noticed the lesion a week back while brushing her teeth. Her father had also noticed that she had excessive salivation and foul odour for the past one month.

Examination revealed a soft inflamed gingival enlargement $(1 \mathrm{~cm} \times 0.5 \mathrm{~cm})$ in relation to the buccal aspect of deciduous right maxillary first molar. Small ulcers (3 in number) were present on the mucosa overlying the swelling. The swelling was non-pulsatile, non-fluctuant and no thrill was elicited. There was also soft food debris in the cervical third of the deciduous right maxillary canine and deciduous right maxillary first molar. There was no mobility of teeth and no alveolar expansion. Bleeding was present on probing. All erupted teeth found to be apparently normal.

Intra oral peri-apical radiograph revealed multiple osteolytic lesions in the region of the deciduous maxillary right canine and first molar. (Figure 1) Further radiograph of the skull (lateral view) revealed multiple punched out osteolytic lesions. (Figure 2) The patient had a normal complete blood cell count with a normal hematocrit, hemoglobin level, and platelet count Her liver function test and coagulation profile were abnormal and an ultrasound scan revealed a metastatic lesion in the liver. Her urine analysis also revealed no significant abnormality. Tissue cytology revealed excessive proliferation of histiocytes suggestive of EG. (Figure 3) Based on clinical, radiographic and histopathology findings a diagnosis of EG was made, and the patient was referred to the regional cancer centre for further treatment. The patient was managed using chemotherapy with vincristine for four weeks. She was also given oral prednisolone for six weeks. Patient is currently normal and is being reviewed both clinically and radiographically at six month intervals.

Case 2: A six year old girl was referred to the outpatient Department of Pedodontics, The patient had consulted a private clinic with a one month history of pain and swelling of the left lower jaw and a day of fever. (Figure 4) There she underwent the removal of badly decayed teeth. Two weeks later she was referred to us as the swelling persisted even after the procedure.

Examination revealed an extra-oral swelling $(1.5 \mathrm{~cm} \mathrm{x} 1.5 \mathrm{~cm})$ on left side of the face obliterating the angle of mandible. The swelling was smooth, firm and nontender. The overlying skin was intact and slightly erythematous. The swelling was non-pulsatile, non-fluctuant and no thrill was elicited. Intra oral examination the region showed a healed extraction site with obliteration of the buccal sulcus. There was also a hard and non fluctuant lesion extending distally from healed extraction site to retromolar pad area. Examination showed no signs of inflammation. An orthopantomograph revealed a multilocular radiolucency with irregular margins. The developing tooth bud of permanent second molar was found to have a floating appearance. (Figure 5) The lesion was found to start at the mid root level of developing first permanent molar. The 
extraction site showed no relevant radiographic abnormality. Her general skeletal radiographic survey was normal.

The patient had a normal blood profile and her liver function test, coagulation profile and urine analysis was normal. Fine needle aspiration cytology showed proliferation of histiocytic cells and infiltration of eosinophils and neutrophils along with increased vascularity. Based on these findings a diagnosis of EG was made, and the patient was referred to the regional cancer centre for further treatment. She was however lost in follow up.

DISCUSSION: EG is a rare disease that affects 5 children per million populations. The peak incidence is from one to four years of age. [2] The second case therefore is a slightly delayed presentation of EG. The most common presenting symptom is local bone pain (41\%). Oral mucosal lesions although infrequent, are characterized by gingival hypertrophy and ulcers of the buccal mucosa, hard and soft palates, and tongue. [3] This rare finding was present in the first case. Extraoral signs and symptoms are quite common. The skull and lower extremities are the most common extraoral sites of bony involvement. [2] This was also evident in the first case. Unifocal single system disease represents about $50 \%$ of maxillofacial EG, with multifocal single system disease and multifocal multisystem disease equally distributed in the remaining $50 \%$ of cases. ${ }^{[4]}$ The unifocal picture was consistent with the second case while the first case showed a multifocal multisystem disease.

EG should be looked for in children presenting with severe bone loss in primary dentition. Other differentials for the same include leukemia, neutropenia, prepubertal periodontitis, hypophosphatasia, fibrous dysplasia, and Papillon-Lefevre syndrome. [5] Acute myeloid leukemia (AML) may present with gingival hypertrophy and appear as EG. Prepubertal periodontitis is essentially periodontitis of primary teeth, and may include gingival inflammation, early loss of primary teeth, and bone loss. Prepubertal periodontitis is usually associated with underlying medical conditions, including but not limited to neutropenia, hypophosphatasia, papillon lefevre syndrome, and acrodynia. However, it can also occur in otherwise healthy children, although they do not suffer from bone or attachment loss. [5] The craniofacial type of fibrous dysplasia in which only the craniofacial complex are involved including the jaw and maxilla. The craniofacial bones are more affected in the polyostotic (50-100\%) than in the monostotic form (20\%). Fibrous dysplasia of the jaws affects the maxilla more frequently than the mandible and affects females more frequently than males. Papillon-Lefevre syndrome is associated with marked destruction of alveolar bone with premature primary tooth loss. This condition is differentiated from EG by the associated hyperkeratosis of the palms and soles. Hypophosphatasia is characterized by low serum alkaline phosphatase levels and excessive excretion of phospho ethanolamine in the urine. The classic oral finding of hypophosphatasia is premature tooth loss generally involving the mandibular primary incisors, which often have abnormally large pulp spaces. [6]

The prognosis of EG as with any other disease depends on early detection. When considering the fact that EG can often present initially with an oral lesion, the knowledge about the oral manifestations can greatly reduce the morbidity and mortality of this disease. Surgical management alone is used in $50 \%$ of cases with an additional $23 \%$ of the lesions being treated with both surgery and radiation therapy. [7] Radiation alone is used in one fifth of affected individuals. Chemotherapy and intralesional steroid injection are the other modes employed. The first case was

Journal of Evolution of Medical and Dental Sciences/ Volume 2/ Issue 23/ June 10, 2013

Page 4128 
successfully managed with chemotherapy. Surgery alone has a $12 \%$ recurrence rate, compared with $25 \%$ for radiation therapy alone and $19 \%$ for combined surgery and radiation therapy. Survival in oral and maxillofacial EG is quite favourable with only $7 \%$ of patients dead from disease. ${ }^{[2]}$ Because future tooth development is often altered or residual defects may remain after the disease has been arrested, orthodontic guidance and prosthetic care may be essential. It is therefore important to prepare and execute a plan to guide the child's growth and development through maturity to adulthood so that the effects of necessary interventions can be reduced to a minimum.

\section{REFERENCES:}

1. Coppes- Zantinga A, Egeler RM. The Langerhans cell histiocytosis X files revealed. Brit J Hematol 2002; 116:3-9.

2. Hicks J, Flaitz CM. Langerhans cell histiocytosis: current insights in a molecular age with emphasis on clinical oral and maxillofacial pathology practice. Oral Surg Oral Med Oral Pathol Oral Radiol Endod. 2005; 100 (2 Suppl):42-66.

3. García de Marcos JA, Dean Ferrer A, Alamillos Granados F, et al. Langerhans cell histiocytosis in the maxillofacial area in adults. Report of three cases. Med Oral Patol Oral Cir Bucal. 2007 Mar 1; 12(2):E145-50.

4. Glotzbecker MP, Carpentier DF, Dormans JP. Langerhans Cell Histiocytosis: Clinical presentation, pathogenesis, and treatment from the LCH etiology research group at the children's hospital of Philadelphia. The University of Pennsylvania Orthopaedic Journal 2002; 15: 67-73.

5. Shafer WG, Hine MK, Levy BM. Oral aspects of metabolic disease. In: textbook of oral pathology 4th Edition: 1997: 633-645.

6. Kamen F, Crespi P, Eisenbud L et al. Papillon-Lefevre syndrome: Pediatric dental management. J Pedodont 1986; 10:356-64

7. Bartnick A, Friedrich RE, Roeser K, et al. Oral Langerhans cell histiocytosis. J CranioMaxillofac Surg 2002; 30:91-6.

8. Senior Lecturer, Department of Pedodontics. Educare Dental College, 


\section{CASE REPORT}

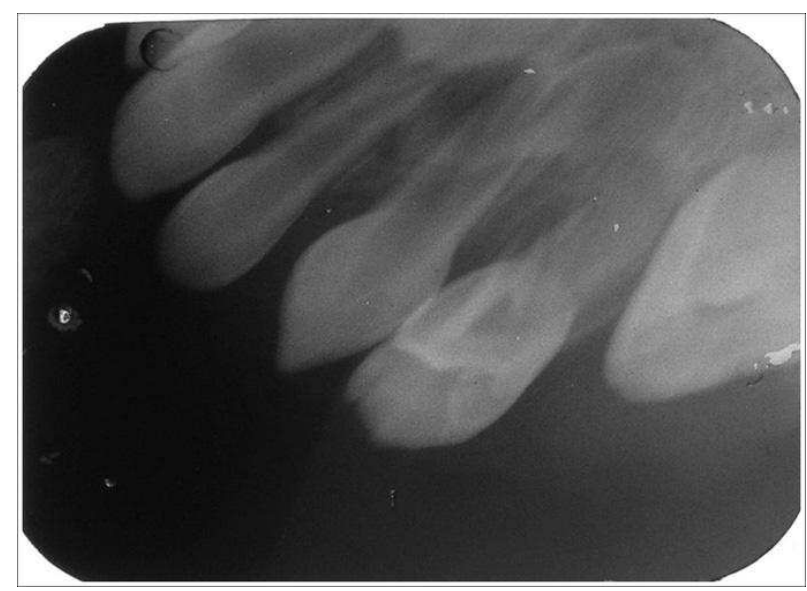

FIGURE 1: IOPA showing punched out lytic lesions in canine and molar regions

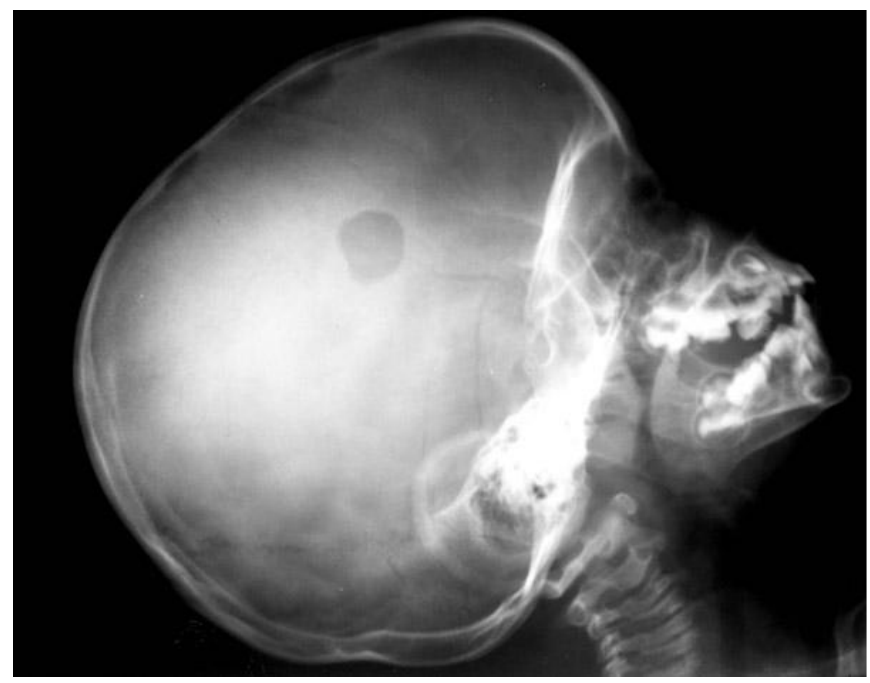

FIGURE 2: Punched out skull lesion

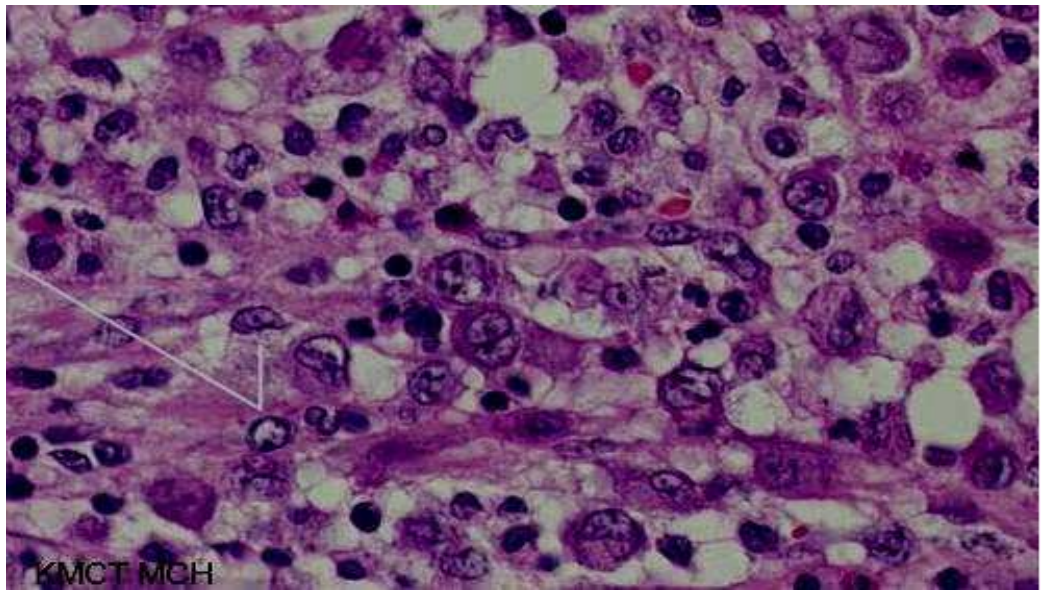

Figure 3: Slide showing excessive proliferation of histiocytes

Journal of Evolution of Medical and Dental Sciences/ Volume 2/ Issue 23/ June 10, 2013 


\section{CASE REPORT}

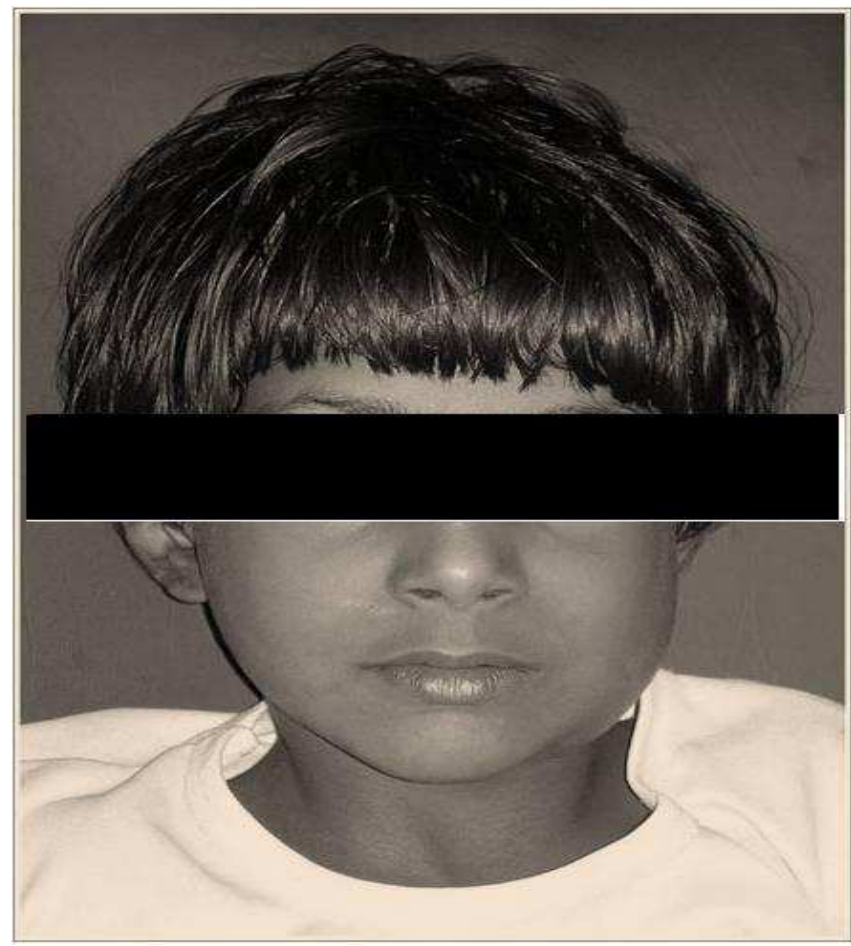

FIGURE 4: Swelling on the left lower jaw

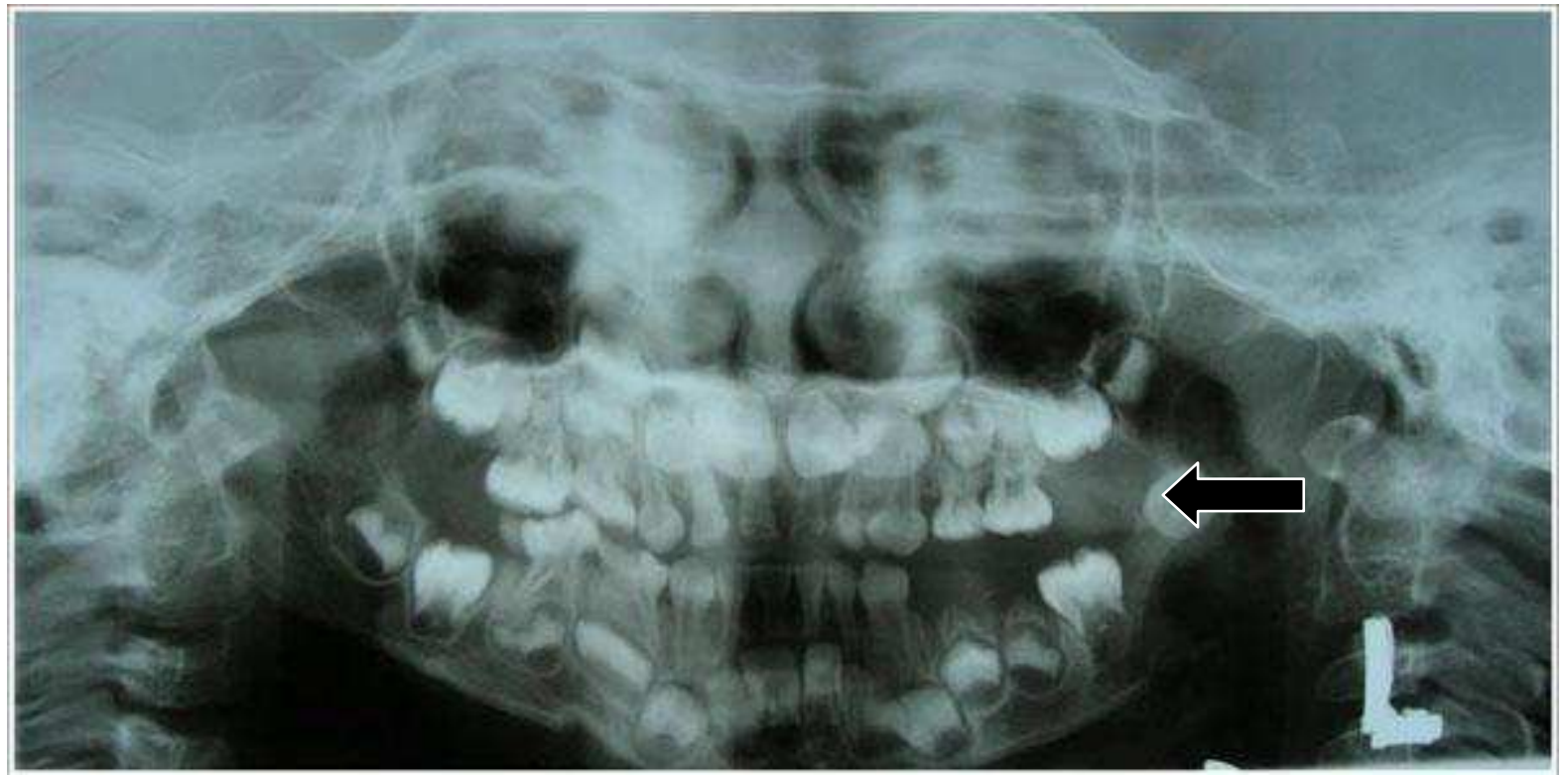

FIGURE 5: OPG showing multilocular radiolucency with irregular margins and floating second molar 